\title{
Quantum wave equations in curved space-time from wave mechanics
}

\author{
Mayeul Arminjon \\ Laboratoire "Sols, Solides, Structures, Risques" (CNRS \& Universités de Grenoble) \\ BP 53, F-38041 Grenoble cedex 9, France. *
}

29 June 2007

\begin{abstract}
Alternative versions of the Klein-Gordon and Dirac equations in a curved spacetime are got by applying directly the classical-quantum correspondence.
\end{abstract}

Key words: quantum mechanics in a gravitational field; Dirac equation.

The usual way to write the wave equations of relativistic quantum mechanics in a curved spacetime is by covariantization: the searched equation in curved spacetime should coincide with the flat-spacetime version in coordinates where the connection cancels at the event $X$ considered. This is connected with the equivalence principle. For the Dirac equation with standard (spinor) transformation, this procedure leads to the Dirac-Fock-Weyl (DFW) equation, which does not obey the equivalence principle. Alternatively, in this work we want to apply directly the classical-quantum correspondence.

${ }^{*}$ Part of this work was done while the author was at Dipartimento di Fisica, Università di Bari and INFN Bari, Italy. 
The latter results [1] from two mathematical facts [2]. i) There is a oneto-one correspondence between a $\left(2^{\text {nd }}\right.$-order, say) linear differential operator:

$$
\mathrm{P} \psi \equiv a_{0}(X)+a_{1}^{\mu}(X) \partial_{\mu} \psi+a_{2}^{\mu \nu}(X) \partial_{\mu} \partial_{\nu} \psi
$$

and its dispersion equation, a polynomial equation for covector $\mathbf{K}$ :

$$
\Pi_{X}(\mathbf{K}) \equiv a_{0}(X)+i a_{1}^{\mu}(X) K_{\mu}+i^{2} a_{2}^{\mu \nu}(X) K_{\mu} K_{\nu}=0,
$$

the latter arising when one looks for "locally plane-wave" solutions [1]: $\psi(X)=$ $A \exp [i \theta(X)]$, with $\partial_{\nu} K_{\mu}\left(X_{0}\right)=0$, where $K_{\mu} \equiv \partial_{\mu} \theta$. The correspondence from (2) to (11) is $K_{\mu} \rightarrow \partial_{\mu} / i$. ii) The propagation of the spatial wave covector $\mathbf{k} \equiv\left(K_{j}\right)(j=1,2,3)$ obeys a Hamiltonian system:

$$
\frac{\mathrm{d} K_{j}}{\mathrm{~d} t}=-\frac{\partial W}{\partial x^{j}}, \quad \frac{\mathrm{d} x^{j}}{\mathrm{~d} t}=\frac{\partial W}{\partial K_{j}} \quad(j=1,2,3),
$$

where $W(\mathbf{k} ; X)$ is the dispersion relation, got by solving $\Pi_{X}(\mathbf{K})=0$ for the frequency $\omega \equiv-K_{0}$. Wave mechanics (classical trajectories=skeleton of a wave pattern) means that the classical Hamiltonian is $H=\hbar W$. The classical-quantum correspondence follows [1] by substituting $K_{\mu} \rightarrow \partial_{\mu} / i$.

This analysis shows [3] that the classical-quantum correspondence needs using preferred classes of coordinate systems: the dispersion polynomial $\Pi_{X}(\mathbf{K})$ and the condition $\partial_{\nu} K_{\mu}(X)=0$ stay invariant only inside any class of "infinitesimally-linear" coordinate systems, connected by changes satisfying, at point $X$ considered, $\frac{\partial^{2} x^{\prime} \rho}{\partial x^{\mu} \partial x^{\nu}}=0, \mu, \nu, \rho \in\{0, \ldots, 3\}$. One such class is that of locally-geodesic coordinate systems at $X$ for metric $\boldsymbol{g}$ : $g_{\mu \nu, \rho}(X)=$ $0, \mu, \nu, \rho \in\{0, \ldots, 3\}$. Another class occurs if there is a (physically) preferred reference frame: that made of changes which are internal to this frame. Assuming one class or the other gives distinct wave equations.

We may now apply this correspondence to a relativistic particle, also in a curved space-time [3]. In each coordinate system, the energy component $p_{0}$ of the 4-momentum defines a classical Hamiltonian $H \equiv-p_{0}$ satisfying

$$
g^{\mu \nu} p_{\mu} p_{\nu}-m^{2}=0 \quad(c=1) .
$$

The dispersion equation associated with this by wave mechanics is

$$
g^{\mu \nu} K_{\mu} K_{\nu}-m^{2}=0 \quad(\hbar=c=1) .
$$


Applying directly the correspondence $K_{\mu} \rightarrow \partial_{\mu} / i$ to it, leads to the KleinGordon equation. Instead, one may try a factorization:

$$
\Pi_{X}(\mathbf{K}) \equiv\left(g^{\mu \nu}(X) K_{\mu} K_{\nu}-m^{2}\right) \mathbf{1}=\left[\alpha(X)+i \gamma^{\mu}(X) K_{\mu}\right]\left[\beta(X)+i \zeta^{\nu}(X) K_{\nu}\right]
$$

Identifying coefficients in (6) (with noncommutative algebra), and then substituting $K_{\mu} \rightarrow \partial_{\mu} / i$, leads to the Dirac equation:

$$
\left(i \gamma^{\mu} \partial_{\mu}-m\right) \psi=0, \quad \text { with } \gamma^{\mu} \gamma^{\nu}+\gamma^{\nu} \gamma^{\mu}=2 g^{\mu \nu} \mathbf{1}
$$

Assume the first class (locally-geodesic systems). Then Eq. (7), derived in any system of that class, rewrites in a general coordinate system as:

$$
\left(i \gamma^{\nu} D_{\nu}-m\right) \psi=0, \quad\left(D_{\nu} \psi\right)^{\mu} \equiv \psi_{; \nu}^{\mu} \equiv \partial_{\nu} \psi^{\mu}+\Gamma_{\sigma \nu}^{\mu} \psi^{\sigma}
$$

(The $\Gamma_{\sigma \nu}^{\mu}$ 's are the Christoffel symbols of $\boldsymbol{g}$.) With the second class (preferred-

frame systems), a different (preferred-frame) equation is got. These two equations are also distinct from the standard, DFW equation.

\section{References}

[1] M. Arminjon, Nuovo Cimento 114B, 71-86 (1999).

[2] G. B. Whitham, Linear and Non-linear Waves (Wiley, New York, 1974).

[3] M. Arminjon, arXiv:gr-qc/0702048 (2007). 